\title{
Determination of the frequency of carbapenemase producing Klebsiella pneumoniae isolates in Dhaka city, Bangladesh
}

\author{
Nawshad Hayder, ${ }^{1,2}$ Zahidul Hasan, ${ }^{2}$ Sadia Afrin ${ }^{1,3}$ and Rashed Noor ${ }^{1 *}$ \\ ${ }^{I}$ Department of Microbiology, Stamford University Bangladesh, 51 Siddeswari Road, Dhaka-1217, Bangladesh; ${ }^{2}$ Department of \\ Microbiology, Ancillary Service, Square Hospital Ltd. 18/F, Bir Uttam Nooruzzaman Sarak, Pantha Path, Dhaka 1205, Bangladesh; \\ ${ }^{3}$ Tuberculosis and Leprosy Department, International Center for Diarrheal Disease Research, Bangladesh (icddr,b), Dhaka 1212, \\ Bangladesh
}

Received 7 September2012/Accepted 2 November 2012

\begin{abstract}
Resistance of Klebsiella pneumoniae against carbapenem, imparted by the presence of carbapenemase, is an emerging global health problem with high morbidity and mortality. Thus, the present study attempted to detect the frequency of carbapenemase producing $K$. pneumoniae in Dhaka city of Bangladesh and thereby determine the health risk associated with their presence. A total of $647 \mathrm{~K}$. pneumoniae isolates were detected from 2800 patients with urinary tract infection, bacterimia, wound infections and respiratory diseases. Thirty one carbapenem resistant isolates were found to harbor $K$. pneumoniae carbapenemase (KPC) through modified Hodge test. The KPC positive isolates were then subjected to the study of antibiogram and showed resistance against all the B-lactam antibiotics along with carbapenems, while they were sensitive against colistin. Additionally, 287 isolates were found to be extended-spectrum $\beta$-lactamases (ESBLs) positive apart from the KPC positive ones.
\end{abstract}

Key words: Carbapenem; Klebsiella pneumoniae carbapenemase (KPC); multi-drug resistance

Enterobacteriaceae, mostly Escherichia coli and Klebsiella pneumoniae, are the most frequent inhabitants among the intestinal microflora causing infections such as cystitis, pyelonephritis, septicemia, pneumonia, peritonitis, meningitis, and device-associated infections (1). They can spread easily among humans and cause community and hospital acquired infections. These microorganisms may acquire resistance against the common antibiotics mostly through plasmids and transposons mediated gene transfer (1-5). In cohort, during the past two decades, extended-spectrum $\beta$ lactamases (ESBLs) producing Enterobacteriaceae has been emerged (6).

$K$. pneumoniae are among the most predominant genera that carry ESBLs and are found to be highly resistant against antibiotics including aminoglycosides, fluoroquinolones, and sulfonamides (6). In order to achieve an effective medication, the use of cephalosporin has increased against the isolates carrying ESBLs. However, in recent years, ESBLs producing isolates, capable of hydrolyzing cephalosporins except carbapenems, have also been reported (1). Therefore, carbapenems are the preferred antibiotic for the treatment of ESBL positive $K$. pneumoniae. Unfortunately, over use of this drug

${ }^{\ddagger}$ Corresponding author. Mailing address: : Dr. Rashed Noor, Department of Microbiology, Stamford University Bangladesh, 51, Siddeswari Road, Dhaka, Bangladesh; Email: noor.rashed@yahoo.com. creates a selective pressure to $K$. pneumoniae strains which resulted in the acquisition of resistance by the production of the enzyme carbapenemase, which destroys carbapenem (7). The emergence of $K$. pneumoniae that harbor the plasmid encoded carbapenemase, commonly known as $K$. pneumoniae carbapenemase (KPC), has now become a global public health concern $(8,9)$.

The prevalence of carbapenemase producers still remains obscure because of the lack of proper detection method in many countries, especially those with limited resources and poor laboratory settings (10). Notably, an early detection of carbapenemase producing bacteria in their primitive infection stage is essential to prevent the progression of hospital-based outbreaks (11). Moreover, the rapid spread of carbapenem resistant $K$. pneumoniae may limit the effectiveness of carbapenems in the treatment of multidrug-resistant infections (12).

Carbapenemases can be detected phenotypically by the modified Hodge test which demonstrate $100 \%$ sensitivity and specificity when compared to other available tests (13, 14). Another standard method of carbapenemase identification based on the detection of specific genes through PCR $(15,16)$. Hence, the eradication of KPC positive $K$. pneumoniae often requires the use of polymixins and tigecycline, even they have been reported to be nephrotoxic (17). The present study aimed to conduct antimicrobial susceptibility of $K$. pneumonia and to determine the prevalence of $K$. pneumoniae harboring KPC in clinical specimens. 


\section{MATERIALS AND METHODS}

The study was carried out at Square Hospital Ltd., Dhaka, Bangladesh from January 2011 to December 2011. Specimens including urine, blood, tracheal aspirates and pus were aseptically collected from hospitalized and out patient reported the hospital. All the specimens were spread over the surface of MacConkey agar, blood agar and chocolate agar media and were incubated at $37{ }^{\circ} \mathrm{C}$ for $18-24$ hours. K. pneumoniae isolates were identified based on their typical colony characteristics followed by biochemical identification tests including the triple sugar iron agar (TSI) test, motility indole urea (MIU) test, citrate utilization test and bile esculin agar test (18).

Detection of extended-spectrum $\beta$-lactamase (ESBL). Isolates were introduced onto Mueller-Hinton agar media. Ceftazidime $(30 \mu \mathrm{g})$, ceftriaxone (30 $\mu \mathrm{g})$, cefotaxime $(30 \mu \mathrm{g})$ and aztreonam $(30 \mu \mathrm{g})$ discs were placed peripherally away from amoxicillin clavulanic acid $(20+10 \mu \mathrm{g})$ disc which was placed at the center of the Mueller-Hinton plate. Band formation between amoxyclav disc and any other disc was considered as ESBL positive (19).

Detection of KPC. Carbapenem (meropenem and imipenem) resistance was determined through antibiotic susceptibility test by disc diffusion method using the Kirby-Bauer technique (20-21). Finally, the detection of KPC from carbapenem resistant isolates was performed by Modified Hodge test (22). A 6 hour culture suspension of E. coli ATCC 25922 adjusted to $0.5 \mathrm{McF}$ arland standard, was introduced on to the surface of Mueller-Hinton agar (MHA) (HIMEDIA, Mumbai, India). After drying, $10 \mu \mathrm{g}$ meropenem disc (HI-MEDIA, Mumbai, India) was placed at the center of the plate and the test strain was streaked from the edge of the disk to the periphery of the plate in four different directions. The plate was incubated overnight at $37{ }^{\circ} \mathrm{C}$. The presence of a 'cloverleaf shaped' zone of inhibition indicated the production of carbapenemase by the test strain.

Study of antibiogram. Antibiotic susceptibility pattern of the carbapenem resistant isolates were then determined (20-21). Meropenem (10 $\mu \mathrm{g})$, imipenem $(10 \mu \mathrm{g})$, cefexime $(5 \mu \mathrm{g})$, cefuroxime $(30 \mu \mathrm{g})$, ciprofloxacin $(5 \mu \mathrm{g})$, ceftriaxone (30 $\mu \mathrm{g})$, cotrimoxazole $(25 \mu \mathrm{g})$, tetracycline $(30 \mu \mathrm{g})$, amikacin $(30 \mu \mathrm{g})$, gentamicin $(10 \mu \mathrm{g})$, nitrofurantoin $(300 \mu \mathrm{g})$, ampicillin $(10 \mu \mathrm{g})$, chloramphenicol $(30 \mu \mathrm{g})$, cefepime $(30 \mu \mathrm{g})$, amoxicillin $(10 \mu \mathrm{g})$, pipercillin tazobactam $(100 \mu \mathrm{g})$ and coloistin $(25 \mu \mathrm{g})$ were used for the assay.

\section{RESULTS}

Determination of the frequency of KPC. A total of $647 \mathrm{~K}$. pneumoniae isolates were included in this study. Among the isolates, $31(4.8 \%)$ isolates were found to be resistant against carbapenems (meropenem and imipenem). All the carbapenem resistant isolates were found to be KPC positive as revealed through the modified Hodge test.

Patients harbouring KPC positive $K$. pneumoniae were under treatment with carbapenem, cephalosporin, vancomycin, linezolid, and combination of carbapenem and cephalosporin. Ages of majority (71\%) of such patients were above 45 years (Table 1). Only two patients $(6.5 \%)$ were below 15 years of age. The proportion of male patients $(58.1 \%)$ were higher over female ones $(41.9 \%)$ (Table 1). Out of 31 patients, 27 had true infections, which were confirmed by observing patient's clinical symptoms and other supportive diagnostic reports. Remaining 4 isolates either colonized or were in unknown state. We found 287 isolates to harbor ESBL which were KPC negative.

Drug-resistance of the KPC positive $K$. pneumoniae isolates. In addition to carbapenems (meropenem and imipenem), all KPC producing isolates were found to be $100 \%$ resistant against cefepime, ceftriaxone, cefexime, cefuroxime, amoxcillin, ciprofloxacillin, ampicillin, nitrofurantoin,
TABLE 1. Age and sex distribution of patients harboring carbapenemase producing $K$. pneumoniae $(\mathrm{n}=31)$

\begin{tabular}{cccc}
\hline Age & Male & Female & $\begin{array}{c}\text { Carbapenemase producing } \\
\text { Klebsiella pneumoniae }(\%)\end{array}$ \\
\hline Up to 15 & 2 & 0 & $2(6.5)$ \\
years & & & $4(12.9)$ \\
15 - 30 years & 4 & 0 & $3(9.7)$ \\
30 - 45 years & 2 & 1 & $8(25.8)$ \\
45 - 60 years & 4 & 4 & $14(45.2)$ \\
Above 60 & 6 & 8 & $31(100)$ \\
years & & & \\
\hline Total $(\%)$ & $18(58)$ & $13(41.9)$ & \\
\hline
\end{tabular}

chloramphenicol and pipercillin-tazobactam (Table 2). Higher resistance was also observed against gentamycin (93.5\%), amikacin (96.8\%), cotrimoxazole (93.5\%) and tetracycline (90.3). Conversely, All KPC positive $K$. pneumeniae were found sensitive against colistin sulphate (Table 2).

TABLE 2. Antibiotic resistance of the KPC positive $K$. pneumoniae isolates $(n=31)$

\begin{tabular}{lc}
\hline Antibiotics $(\mu \mathrm{g})$ & Number of resistant isolates $(\%)$ \\
\hline Meropenem (10) & $31(100)$ \\
Imipenem (10) & $29(100)$ \\
Gentamycin (10) & $30(96.8)$ \\
Amikacin (30) & $31(100)$ \\
Cefepime (30) & $31(100)$ \\
Ceftriaxone (30) & $31(100)$ \\
Cefexime (10) & $31(100)$ \\
Cefuroxime (30) & $31(100)$ \\
Amoxcillin (10) & $31(100)$ \\
Ciprofloxacillin (5) & $31(100)$ \\
Nitrofurantoin (300) & $28(90.3)$ \\
Tetracyclin (30) & $31(100)$ \\
Chloramphenicol (30) & $29(93.5)$ \\
Cotrimoxazole (10) & $0(0)$ \\
Colistin (25) & $31(100)$ \\
Ampicillin (10) & $31(100)$ \\
Pipercillin Tazobactam (100)
\end{tabular}

\section{DISCUSSION}

The emergence and rapid dissemination of KPC producing $K$. pneumoniae is now a global health threat $(23,24)$. Such multi-drug resistant bacteria are difficult to treat as the treatment options are limited. The delay in microbiological laboratory confirmatory results often occur due to limitation in laboratory detection (23). Therefore, infection by KPC may accounts for an increase in the rate of morbidity and mortality of patients compared to infection by carbapenem susceptible Enterobacteriaceae (25). Importance of carbapenemaseproducing pathogens underlies the fact that the genes 
coding for KPC enzymes are on plasmids that can transmit resistance between organisms and between species $(6,26)$. Therefore, healthcare facilities must be vigilant in maintaining good infection control practices.

As revealed from the present study, out of $647 \mathrm{~K}$. pneumoniae isolates, $4.79 \%$ were found to be resistant against carbapenems and hence were KPC positive, which is 3 times higher than that found in India, where only $1.70 \%$ KPC positive $K$. pneumoniae isolates were identified (27). The elevated frequency of KPC positive isolates in present study might be due to the increased use of carbapenem in Dhaka, Bangladesh as compared to India. Out of $31 \mathrm{KPC}$ positive isolates, 27 $(87.1 \%)$ showed clinical manifestation. In this study, clinical history of patients harboring KPC positive $K$. pneumoniae revealed that most patients had prior and/or prolonged hospitalization, ICU stay, and/or prior antibiotic therapy. Interestingly, from our study, none of the KPC positive isolates found to harbor ESBLs. This finding indicated that carbapenem might be the drug of choice for the treatment of ESBL positive isolates.

The antibiotic susceptibility patterns of KPC positive isolates revealed that the isolates were highly resistant against most of the antibiotics used except for colistin. Therefore, we endorse colistin to be the drug of choice for the treatment of KPC positive isolates. In another study it was found that KPC positive patients were successfully treated using tigecycline (28). However, in the present study, the effectiveness of trigecycline against KPC positive isolates was not tested.

\section{ACKNOWLEDGEMENTS}

We thank Square Hospital Ltd., Dhaka, Bangladesh for the technical facilities and Stamford University Bangladesh for the financial support.

\section{REFERENCES}

1. Nordmann, P., T. Naas, and L. Poirel. 2011. Global Spread of Carbapenemase producing Enterobacteriaceae. Emer Infect dis. 17 (10): 1791-1798.

2. Canton, R. 2008. Antibiotic resistance genes from the environment: A perspective through newly identified antibiotic resistance mechanisms in clinical setting. European Soc Clin Microbiol Infect Dis 15: 20-25.

3. Hung D. T. and B. B. Kaufman. 2010. The Fast Track to Multidrug Resistance. Mol Cell 37: 297-298.

4. Ochiai, K., T. Yamanaka, K. Kimura, and O. Sawada. 1959. Inheritance of drug resistance (and its transfer) between Shigella strains and Between Shigella and E. coli strains. Hihon Iji Shimpor 34: 1861.

5. Bennett, P. M. 2008. Plasmid encoded antibiotic resistance: Acquisition and transfer of antibiotic resistance genes in bacteria. Br J Pharmacol 153: $347-357$.
6. Bradford, P. A., et al. 2004. Emergence of Carbapenem-Resistant Klebsiella Species Possessing the Class A Carbapenem-Hydrolyzing KPC-2 and Inhibitor-Resistant TEM-30 $\beta$-Lactamases in New York City. Clin. Infect. Dis. 39 (1): 55-60.

7. Coque, T. M., F. Baquero, and R. Canton. 2008. Increasing prevalence of ESBL producing Enterobacteriaceae in Europe. Eurosurveillance 13 (47): 1929.

8. Ibeawuchi, R. and T. I. Mbata. 2002. Rational and irrational use of antibiotics. African Health 24 (2): 16-18.

9. Fontana, C., et al. 2010. Emergence of KPC producing Klebsiella pneumoniae in Italy. BMC Research Notes 3: 40 .

10. Jean, S. S. and P. R. Husueh. 2011. High burden of antimicrobial resistance in Asia. Int J Antimicrob Agents 37: 291-295.

11. Falagas, M. E., D. E. Karageorgopoulos, and P. Nordmann. 2011. Therapeutic options with Enterobacteriaceae producing carbapenemhydrolyzing enzymes. Future Microbiol 6: 653-656.

12. Manchanda, R., et al. 2011. Survey for carbapenemase-producing Klebsiella pneumoniae isolated from clinical specimens in thai hospitals. J Infect Dis Antimicrob Agents 28: 242-243.

13. Lee, K., et al. 2011. Modified Hodge and EDTA-disk synergy tests to screen metallo- $\beta$-lactamase-producing strains of Pseudomonas and Acinetobacter species. Clin Microbiol Infect. 7: 88-91.

14. Doi, Y. 2008. Simple disk-based method for detection of Klebsiella pneumonia carbapenemase-typ $\beta$-lactamase by use of a boronic acid compound. J Clin Microbiol 46 (12): 4083-4086.

15. Miriagou, V., et al. 2010. Acquired carbapenemases in Gram-negative bacterial pathogens: detection and surveillance issues. Clin Microbiol Infect 16: $112-22$.

16. Nordmann, P., et al. 2011. How to detect NDM-1 producers. J Clin Microbiol 49: 718-21

17. Kanj, S. S. and Z. A. Kanafani. 2011. Current concepts in antimicrobial therapy against resistant gram-negative organisms: extended-spectrum $\beta$ lactamase-producing Enterobacteriaceae, carbapenem-resistant enterobacteriaceae, and multidrug-resistant Pseudomonas aeruginosa. Mayo Clin Proc 86 (3): 250-259.

18. Collee, J. G., R. S. Miles, and B. Watt. 1996. Tests for the identification of bacteria, p. 131-145. In Collee, J. G., B. P. Marmion, and A. Simmons (Eds.), Practical Medical Microbiology. Churchill Livingstone, London.

19. Jarlier, V., M. H. Nicolas, G. Fournier, and A. Philippon. 1998. ESBLs conferring transferable resistance to newer beta lactam agents in Enterobacteriaceae: hospital prevalence and susceptibility patterns. Rev Infect Dis. 10: $867-878$.

20. Bauer, A. W., W. M. M. Kirby, J. C. Sheriss, and M. Turck. 1966. Antibiotic susceptibility testing by standardized single disc method. Am J Clinical pathol 45: 493-496.

21. Ferraro, M. J., W. A. Craig, and M. N. Dudley. 2001. Performance standards for antimicrobial susceptibility testing., 11th ed. NCCLS informational supplement, Pennsylvania, USA

22. Pasteran, F., et al. 2009. Sensitive screening tests for suspected class A carbapenemase production in species of Enterobacteriaceae. J Clin Microbiol 47 (6): 1631-1639.

23. Arnold, R. S., et al. 2011. Emergence of Klebsiella pneumoniae carbapenemase (KPC)-producing Bacteria. South Med J 104 (1): 40-45.

24. Queenan, A. M. and K. Bush. 2007. Carbapenemases: the versatile $\beta$ lactamases. Clin Microbiol Rev. 20: 440-58.

25. Center for Disease Control (CDC). 2009. Guidance for control of infections with carbapenem-resistant or carbapenemase-producing Enterobacteriaceae in acute care facilities. MMWR 58 (10): 256-260.

26. Yigit, H., et al. 2001. Novel carbapenem-hydrolyzing beta-lactamase, KPC-1, from a carbapenem-resistant strain of Klebsiella pneumoniae. Antimicrob Agents Chemother. 45: 1151-61.

27. Kumarasamy, K. K., et al. 2010. Emergence of a new antibiotic resistance mechanism in India, Pakistan, and the UK: a molecular, biological, and epidemiological study. Lancet Infect Dis 10: 597-602

28. Daly, M. W., et al. 2007. Tigecycline for treatment of pneumonia and empyema caused by carbapenemase-producing Klebsiella pneumoniae. Pharmacotherapy 27: 1052-1057. 